\title{
PERSPECTIVE
}

\section{Testosterone replacement therapy in the era of telemedicine}

\author{
Justin M. Dubin $\mathbb{D}^{1 凶}$, Richard J. Fantus $\mathbb{D}^{2}$ and Joshua A. Halpern ${ }^{2}$ \\ (c) The Author(s), under exclusive licence to Springer Nature Limited 2021
}

The events of the 2019 SARS-CoV2 virus pandemic have all but ensured that telemedicine will remain an important aspect of patient care delivery. As health technologies evolve, so must physician practices. Currently, there is limited data on the management of testosterone replacement therapy (TRT) in the era of telemedicine. This review aims to explore the potential benefits and pitfalls of TRT management via telemedicine. We also propose a theoretical framework for TRT management via telemedicine. Telemedicine provides patients and physicians with a new mechanism for American Urological Association guidelineconcordant TRT management that can increase patient access to care and provide a safe space for men who may otherwise not have been comfortable with in-person evaluation. However, there are significant limitations to the use of telemedicine for the management of TRT, including the inability to perform a physical exam, inability to administer specific medications, technological barriers, data security, and medical-legal considerations, and both patients and providers should engage in shared decision making before pursuing this approach. Understanding and acknowledging the potential pitfalls of telemedicine for TRT management will enable both patients and providers to achieve optimal outcomes and satisfaction.

IJIR: Your Sexual Medicine Journal (2022) 34:663-668; https://doi.org/10.1038/s41443-021-00498-5

\section{INTRODUCTION}

Telemedicine is the use of electronic communications to provide patients with medical services without an in-person visit. Even prior to the 2019 SARS-CoV2 virus (COVID-19) pandemic, the use of telemedicine had increased substantially across healthcare delivery systems. By 2018, growth projections estimated that the telemedicine industry would comprise a $\$ 48.8$ billion market by 2023 [1]. This growth has been accelerated by COVID-19, which has led to an even more rapid adoption of telemedicine, as demonstrated by a nearly three-fold increase in telemedicine usage by urologists [2]. As a result, the American Urological Association (AUA) recently updated its white paper on telemedicine in urology, concluding that urologists should continue to embrace telemedicine, as it will likely remain a critical and permanent element in health care delivery and urologic training [3].

Direct to consumer (DTC) online medical platforms represent a specific approach towards telemedicine, and these DTC platforms have seen exponential growth in the past few years, especially in the field of urologic care [4]. Focusing predominantly on men's health issues such as erectile dysfunction (ED), hair loss, and male infertility, these online companies perform basic healthcare screenings and then prescribe medications without physical examinations [5, 6]. Even prior to COVID-19, these platforms were growing in popularity among men in the United States (US). From 2017 to 2019, there was a 1500\% increase in online visits to DTC platforms treating ED with over 11 million visits to these DTC sites in the fourth quarter of 2019 alone [4]. The widespread popularity of these platforms, in conjunction with the embrace of telemedicine by influential medical organizations such as the AUA, suggests that telemedicine for men's health is here to stay.
Testosterone replacement therapy (TRT) is likely the next frontier for telemedicine in men's health. In recent years, the use of TRT has increased four-fold in men aged 18-45 years and three-fold in older men [7]. Testosterone deficiency (TD) and treatment with TRT is considered within the scope of men's health, and as such, it is expected that popular DTC men's health platforms will begin to offer evaluation and treatment of TRT in the near future. Indeed, a handful of small, start-up initiatives in this space are already offering DTC care for this condition. Moreover, as urologists continue to embrace and adapt telemedicine in day-to-day clinical practice, a significant proportion of TRT care delivery may shift towards remote care delivery, as TD is a clinical condition highly amenable to management through telemedicine.

Currently, there is limited data on the management of TRT in the era of telemedicine. This review aims to explore the potential benefits and pitfalls of TRT management via telemedicine. We also propose a theoretical framework for TRT management via telemedicine.

\section{THE BENEFITS}

Telemedicine offers a multitude of benefits for both patients and care providers. These include improved access to care, patient and physician flexibility, decreased costs, and potential for destigmatization of care (Table 1) [8]. Telemedicine also has a clear role in the reduction of infectious disease transmission, which largely accounts for the rapid increase in utilization during the COVID-19 pandemic.

\section{Access to care}

A critical advantage of telemedicine is its ability to broaden access to subspecialty men's health care for a wide number of patients. 
Table 1. Advantages and disadvantages of telemedicine for the management of testosterone deficiency (TD) and testosterone replacement therapy (TRT)

$\begin{array}{ll}\text { Advantages } & \text { Disadvantages } \\ \uparrow \text { Access to care } & \text { Lack of a physical exam } \\ \begin{array}{l}\uparrow \text { Convenience for patients/ } \\ \text { providers } \\ \text { costs }\end{array} & \begin{array}{l}\text { Inability to administer } \\ \text { medication }\end{array} \\ \begin{array}{l}\uparrow \text { Satisfaction for patients/ } \\ \text { providers }\end{array} & \text { Dechnological barriers to access } \\ \begin{array}{l}\downarrow \text { Transmission of infectious } \\ \text { disease }\end{array} & \text { Medical-legal liability } \\ & \text { Physician reimbursement }\end{array}$

Geographic barriers to specialty care are abundant, particularly for patients living in rural areas. Nangia et al. previously found that there were less than $\mathbf{2 0 0}$ male infertility specialists in the entire US, and a large number of patients resided more than a 60-min drive from the nearest specialist [9]. While the number of specialists offering care for TD is likely substantially greater, as it includes both reproductive urologists and endocrinologists, there are likely a large number of men without geographic proximity to a specialist with expertise in the management of TD. Telemedicine enables men who may not readily have access to fellowshiptrained andrologists and endocrinologists to establish subspecialty care.

Broadening access to high-quality, subspecialty care for TD through telemedicine is particularly important in the setting of continued proliferation of for-profit, men's health clinics. The dramatic increase in testosterone prescriptions and sales over the past few decades has been attributed, in part, to the rise of these for-profit "low T" clinics, where TRT is often prescribed without clear indications and without appropriate monitoring [10]. Many of these clinics offer fee-based membership plans for TRT and offer only limited treatment options for hypogonadal men, potentially depriving patients of alternative treatment options. The wider accessibility of fellowship-trained specialists through telemedicine can help to ensure that patients receive safe, guideline-concordant, subspecialty care and avoid these forprofit "low T" clinics.

Telemedicine not only facilitates new patient consultations with subspecialists managing TRT, but it also eases the burden of follow-up visits for patients who have already established care. According to the AUA guidelines on the Evaluation and Management of Testosterone Deficiency, follow-up appointments are typically recommended initially $2-4$ weeks after starting TRT and subsequently every 6-12 months [11]. These appointments are important to ensure adequate therapeutic response and patient safety while on TRT. Frequent follow-up can be burdensome for patients, especially if it requires taking time off work and traveling long distances to be seen in person, which can lead to inappropriate follow-up or discontinuation of therapy [12]. The ease of scheduling and completing telemedicine follow-up visits could reduce the number of men with delayed follow-up, loss of follow-up, or discontinuation of therapy due to these barriers.

\section{Overcoming stigma associated with men's health}

An increasingly important aspect of men's health involves the destigmatization of men seeking medical care. Multiple studies have found that perceived stigma in seeking and receiving care is a major barrier in treating men's health issues [13-16]. Signs and symptoms of TD include reduced energy, reduced endurance, loss of body hair, reduced beard growth, reduced muscle mass, depressive symptoms, reduced sex drive, and reduced erectile function [11]. These symptoms can lead to health-related self-stigma, which can make it difficult for men to discuss these issues with a provider [14-16]. Telemedicine provides an avenue for men who may feel uncomfortable traveling to a doctor's office to discuss their health issues in a more private setting.

\section{Provider flexibility}

Another major benefit of telemedicine is that it provides flexibility for the physician [8]. Physician burnout has been increasingly recognized as a pervasive issue throughout the US healthcare system. Burnout is a major concern specifically among urologists, with whom symptoms are expressed by more than $50 \%$ of providers $[17,18]$. One of the major drivers of burnout includes work-home conflicts [19]. Through the use of telemedicine, providers are no longer forced to practice exclusively in the office-this affords the opportunity to practice safe, effective, and guideline-appropriate patient care from the location of their choice. It is possible that provider flexibility will not only allow for satisfactory care, but also reduce the risk of physician burnout.

\section{Decreased costs}

The use of telemedicine is associated with reduced costs for both patients and providers. Demaerschalk et al. [20] performed a health economic analysis examining costs associated with inperson versus telehealth visits at a large, integrated practice. They considered costs of travel, accommodations, meals, and missed work, finding that telemedicine visits were estimated to save $\$ 888$ per visit, on average. While these savings were predominantly driven by patients living very far away from the primary practice location, the study demonstrates the financial upside of telemedicine for select patients. Likewise, Zholudev et al. examined the use of telemedicine among veterans with substantially shorter travel distances and found modest but significant savings of $\$ 124$ for each virtual encounter [21].

Provider cost savings may also be associated with increased use of telemedicine, though specific data in urology and TRT are lacking [22]. While the implementation of telemedicine can require initial capital investment from providers and practices, subsequent use of telemedicine can theoretically deliver longterm cost savings through decreased resource utilization. Portney et al. [23] utilized time-driven activity-based costing to quantify costs associated with telemedicine and in-person visits across two urological surgery clinics and found that physician-led video visits did not offer cost savings compared to in-person visits, whereas physician assistant-led video visits were significantly less costly. Further data are needed to determine the long-term cost-saving potential of telemedicine, specifically in the setting of TRT.

\section{Patient and provider satisfaction}

In aggregate, these numerous benefits of telemedicine have led to high satisfaction rates for both patients and providers. Ramaswamy et al. [24] analyzed Press Ganey patient satisfaction surveys from over 38,000 patients including 620 video visits and found that satisfaction scores were significantly higher for video visits compared to in-person visits $(94.9 \%$ vs $92.5 \%, P<0.001)$. Studies across multiple subspecialty disciplines within urology including urologic oncology, pediatric urology, neuro-urology, and female pelvic and reconstructive medicine have demonstrated high satisfaction rates with telemedicine during the pandemic [2529]. Most recently, Shiff et al. examined patient satisfaction rates in a single andrology clinic and found that the majority of patients were satisfied with a telephone-mediated visit as a substitute for an in-person visit during the COVID-19 pandemic. However, $27.1 \%$ of patients preferred an in-person visit, indicating that telemedicine may not be the optimal approach for a significant minority of andrology patients [30]. While additional data are needed to evaluate patient satisfaction with the use of telemedicine for TRT 
specifically, the above-mentioned data suggest the potential for high satisfaction.

While much literature has focused on patient satisfaction with telemedicine, multiple studies have demonstrated that providers are, likewise, highly satisfied with telemedicine. The Telehealth Impact Study, a nationwide survey of over 1500 providers, revealed high satisfaction rates with telemedicine, which is also reflected in the limited urological literature [31, 32].

\section{THE POTENTIAL PITFALLS}

Despite its benefits, telemedicine for TRT does have several drawbacks. These shortcomings include the inability to perform a physical exam, the inability to administer specific medications, and more general limitations pertaining to technological barriers, data security, and medical-legal considerations (Table 1) [33-35].

\section{Lack of physical examination}

A critical limitation of telemedicine is the inability to perform a complete, hands-on physical examination. Men with TD may present with a variety of physical signs and symptoms including changes in hair growth, muscle mass, and body fat that can be detected on physical examination [36]. Men with TD are also likely to have significant underlying medical comorbidities that may require a physical exam for diagnosis $[37,38]$. For example, undiagnosed comorbidities such as cardiovascular disease can be missed without the vital sign, cardiopulmonary or lower extremity examination $[39,40]$. Furthermore, men with TD may have related, treatable pathologies such as erectile dysfunction, which may also require a physical exam to assess for concurrent pathology such as Peyronie's disease in addition to the aforementioned comorbidities [10, 41, 42]. Lastly, a significant number of men receiving TRT are between the age of 55-69, the optimal age for prostate cancer screening. The AUA recommends that all men over the age of 40 receiving TRT should undergo prostate-specific antigen (PSA) measurement to exclude a diagnosis of prostate cancer prior to commencement of therapy, and telemedicine does not facilitate the performance of a digital rectal examination (DRE), historically considered a cornerstone of prostate cancer screening [43].

Interestingly, the AUA guidelines do not specifically mandate a physical examination for the diagnosis and treatment of TD. According to the AUA, the initial evaluation for TD must include the assessment of symptoms and/or signs of TD and a minimum of two total AM testosterone levels that are less than $300 \mathrm{ng} / \mathrm{dl}$ [11]. Therefore, it is possible to evaluate and manage patients interested in TRT via telemedicine and provide guideline-based care. Likewise, while digital rectal examination (DRE) has historically been an essential component of prostate cancer screening, which is required prior to initiation of TRT, neither the AUA guideline on TD nor the AUA guideline on Early Detection of Prostate Cancer mandate DRE $[11,44]$. Thus, it is possible to provide guideline-based care for initiation and management of TRT through telemedicine alone, without a physical examination.

Inability to administer medication and manage complications TRT can be administered in a wide range of formulations, some of which require in-person teaching or administration by a healthcare provider. Men performing intramuscular injections may require an in-person demonstration by a healthcare provider prior to initiation of self-injection therapy, and some men without the required physical dexterity may require in-person administration of the medication on an ongoing basis. All men opting for testosterone pellet therapy require a minimally invasive, in-office procedure for administration. The use of telemedicine alone for TRT management may limit these therapeutic options.

Lack of in-person care may also lead to difficulty in the management of complications related to TRT use. For example, men who suffer allergic reactions from TRT formulations or those who develop local complications (ex: hematoma or infection following intramuscular injection) may not have a local provider for urgent evaluation [45]. These men may present to local urgent care or primary care providers, which could lead to fragmented, higher-cost care.

\section{Technological barriers}

The use of telemedicine requires that men have access to the necessary technology and be facile in using it. Andino et al. [46] found that $94 \%$ of patients visiting an outpatient urology clinic had access to a device that could be used for a video visit, indicating that only a small minority did not. However, owning a device does not necessarily imply an ability to use that technology for telemedicine. Men initiating TRT tend to be older (median age 71 years), and these men are more likely to be affected by the digital divide - the inability to access or readily use technology [47]. The digital divide disproportionally affects men with disabilities, non-Caucasian men, men with a lower socioeconomic status, and men who live in rural areas [34, 48, 49]. For these patients, using technology to conduct virtual visits introduces non-medical elements of complexity, which can negatively influence their visits. Margolin et al. [26] noted that $9 \%$ of patients utilizing telemedicine for genitourinary cancer care encountered technological barriers, which translated into lower satisfaction rates for both patients and physicians in these instances. These technological difficulties lead to erosion of rapport between patient and physician, which has been a significant complaint among physician users of telemedicine [50].

\section{Data security}

Data theft cost the healthcare industry nearly 4 billion dollars in 2019 [51]. While telehealth has rapidly expanded, in part due to COVID-19, cybersecurity has not increased at the same rate [35]. There are numerous opportunities and entry-points for hackers and bad actors to access and steal patient information including the patient's computer or cellular phone, the patient's router, central data processing hubs, health information repositories or at the level of the provider accessing the patient's data (Fig. 1) $[35,52,53]$. While the exact risk of a data breach with each telemedicine encounter is not precisely known, the threat of compromised protected health information alone may undermine the patient-physician relationship.

\section{Medical-legal considerations}

The introduction of telemedicine has also raised new medical-legal considerations. Interstate licensing was a major barrier to widespread telemedicine use prior to COVID-19, but these laws were relaxed during the pandemic [54]. Despite some relaxation of licensing issues across state lines, licensing requirements vary between states and different rules apply regarding what constitutes telemedicine and which patients are eligible to receive it [55]. Many practitioners are concerned that telemedicine generally exposes physicians to a new form of medical malpractice, but this has yet to be determined [56, 57].

There are also medical-legal concerns regarding the prescription of controlled substances to patients who have never been seen in person. All TRT formulations are considered Schedule III substances according to the US Food and Drug Administration, and these substances also carry unique warning labels regarding the risks of therapy [58]. In line with telemedicine laws, requirements for online prescribing of controlled substances vary by state, and out-of-state prescribers may have additional regulations [55]. While many of these medical-legal considerations have yet to be elucidated or resolved, as telemedicine use continues to increase in the coming years, there will likely be improved clarity and guidance regarding the medical-legal implications of telemedicine use. 


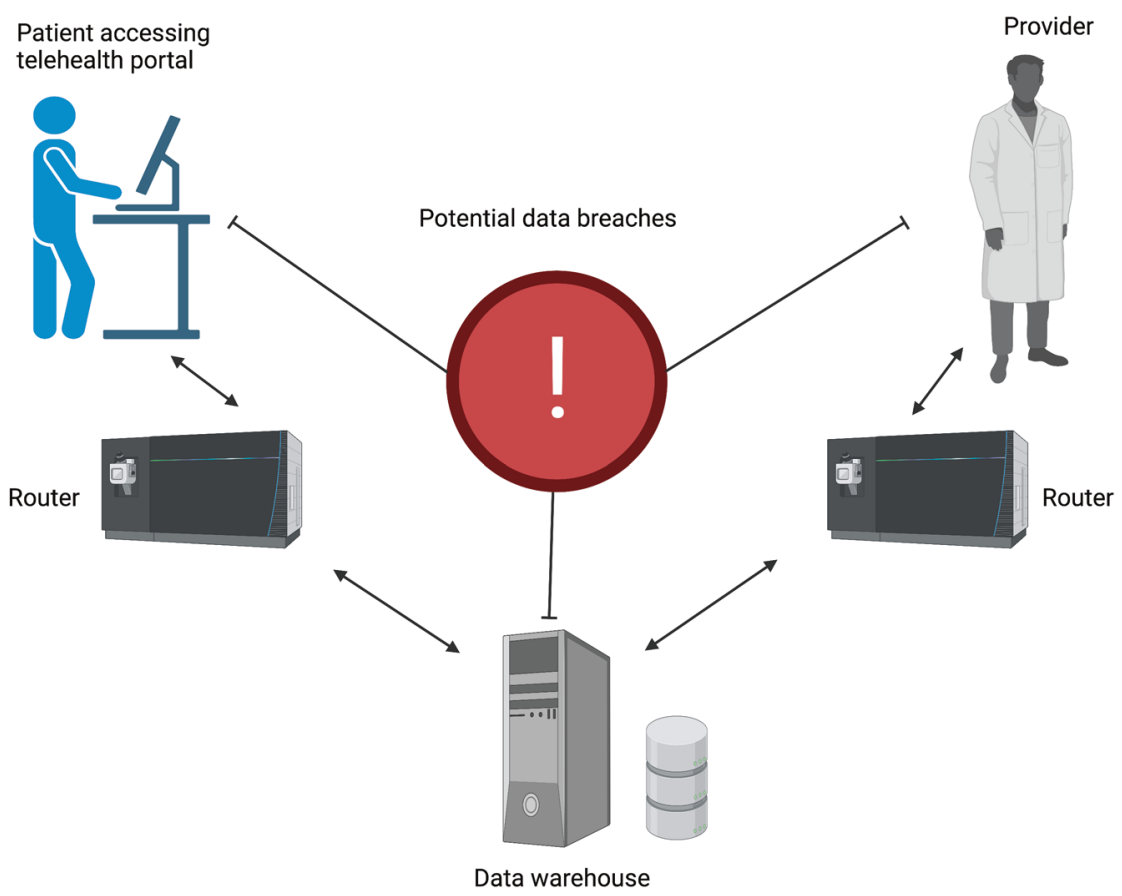

Fig. 1 Potential avenues for data security breach. The use of telemedicine for management of testosterone deficiency (TD) and administration of testosterone replacement therapy (TRT) via telemedicine has several potential avenues for data securtiy breach. (Created with BioRender.com).

At the time of this publication, the legislative landscape impacting the practice of telemedicine in the US is complex. After the initial emergency expansion of telehealth during the COVID-19 pandemic, the legislative protection for continued expansion of telehealth services is currently in jeopardy. Recently, the Telehealth Modernization Act was introduced in Congress, a bill that would permanently expand the telemedicine services initially permitted during the COVID-19 emergency. However, the bill has not yet passed, and even with its passage, a number of legislative issues pertaining to telemedicine, cross-state prescribing, and conflicts between federal and state laws persist. As such, we recommend that any physician providing care through telehealth become familiar with these legislative issues and as they pertain to the physician's unique practice location and environment.

\section{Physician reimbursement}

Physician reimbursement for services rendered is a major consideration when utilizing telemedicine. Technology is constantly evolving and so must coding and physician reimbursement measures. Prior to COVID-19, a major barrier to telemedicine adoption was the concern among providers regarding inferior reimbursement for care provided through telehealth. During the pandemic, the Centers for Medicare and Medicaid Services (CMS) expanded and updated its reimbursement for telehealth services, alleviating some of these concerns [59]. Nonetheless, inconsistencies among payers and states in reimbursement schedules for telemedicine remain a major issue, and many providers lack clarity regarding the specific reimbursement schedules applicable to their practice [60]. In order for telemedicine to remain a mainstay of urologic care, further clarity and expanded reimbursement will be necessary.

\section{PROPOSED WORK-FLOW FOR TRT MANAGEMENT VIA TELEMEDICINE}

Despite the aforementioned limitations, telemedicine offers great potential in the management of TD and TRT for specific patients and providers. However, there is a lack of data and guidance on the proper usage of telemedicine for TRT. Here, we propose a theoretical workflow for the appropriate use of telemedicine in the management of patients on TRT, informed by a thorough review of the literature (Fig. 2).

We recommend that the initial evaluation of men with suspected TD should be performed in-person and by the prescribing physician, if possible. While the AUA does not mandate physical examination in the initial evaluation of TRT, an in-person visit will provide the prescribing physician with the opportunity to perform a physical examination and establish a good rapport with the patient. If an in-person exam with the prescribing physician is not possible, we recommend that the patient be examined in-person by their local primary care provider (PCP) in conjunction with the initial telehealth consultation. Although telemedicine alone is adequate for practicing within the AUA guidelines, an exam by a PCP can help overcome some of the potential pitfalls of telemedicine and can ensure the patient has established primary care in his local community for the management of future issues or complications. Shared decisionmaking should be performed at the time of initial consultation regarding the indications for, risks and benefits of initiation of TRT itself, as well as the risks and benefits of the telemedicine approach to TRT management. Of note, many men may choose not to pursue a telehealth approach to TRT management, and men opting for specific TRT administrations (ex: testosterone pellets) will not be eligible for exclusive telemedicine follow-up.

After the initial visit, follow-up appointments for TRT should be performed via telemedicine, ideally through video visits, for all men opting for a telehealth care pathway. For men continuing on the telemedicine pathway, routine laboratory evaluation, as recommended by the AUA, should be performed prior to each appointment and should be available for physician review [11]. Patients are required to have follow-up every 6 to 12 months for TRT. These frequent visits include an evaluation of lab work and a signs and symptoms check-appointments that are perfectly designed and appropriate for telemedicine. The use of formal, validated questionnaires is not recommended in the management 


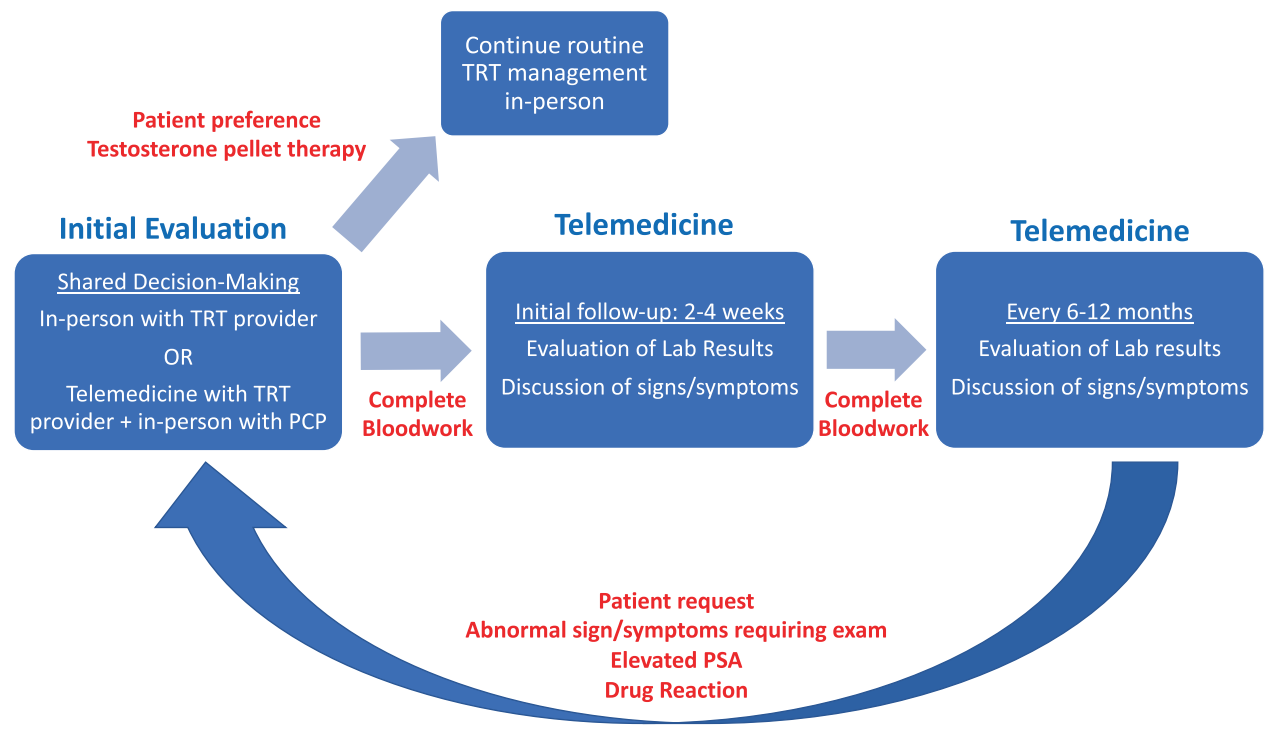

Fig. 2 Proposed workflow for the management of testosterone deficiency (TD) and administration of testosterone replacement therapy (TRT) via telemedicine (created with BioRender.com).

of TD, and as such, we recommend that history taking and symptom evaluation should be performed according to the clinician's typical practice [11].

Telemedicine appointments can continue indefinitely unless otherwise indicated. Indications prompting a return to an inperson appointment include a patient request or any patient sign or symptom that requires a physical exam, which can be performed by either the TRT provider or a local primary care physician, depending upon the clinical scenario.

\section{CONCLUSION}

The events of the COVID-19 pandemic have all but ensured that telemedicine will remain an important aspect of patient care delivery. As health technologies evolve, so must physician practices. The management of TRT via telemedicine provides patients and physicians with a new mechanism for AUA guidelineconcordant TRT management that can increase patient access to care and provide a safe space for men who may otherwise not have been comfortable with in-person evaluation. However, there are significant limitations to the use of telemedicine for the management of TRT, and both patients and providers should engage in shared decision-making before pursuing this approach. Understanding and acknowledging the potential pitfalls of telemedicine for TRT management will enable both patients and providers to achieve optimal outcomes and satisfaction.

\section{REFERENCES}

1. PSM Research. Telemedicine market to cross $\$ 48.8$ billion by 2023: P\&S Market Research https://www.psmarketresearch.com/. 2018.

2. Dubin JM, Wyant WA, Balaji NC, Ong WL, Kettache RH, Haffaf M, et al. Telemedicine usage among urologists during the COVID-19 pandemic: crosssectional study. J Med Internet Res. 2020;22:e21875.

3. Gettman M, Kirshenbaum E, Rhee E, Spitz A. Workgroup AT AUA White paper: telemedicine in urology. Baltimore, MD: America Urological Association; 2021.

4. Wackerbarth JJ, Fantus RJ, Darves-Bornoz A, Hehemann MC, Helfand BT, Keeter MK, et al. Examining online traffic patterns to popular direct-to-consumer websites for evaluation and treatment of erectile dysfunction. Sex Med. 2021;9:100289.

5. Pew Research Center. The internet and health. 2013. https://www.pewresearch. org/internet/2013/02/12/theinternet-and-health/.

6. Clavijo R, Ramasamy R, Halpern J, Melnick A, Stewart J, Rosenwaks Z, et al. "Online" and "at-home" versus traditional models of health care: enhancing access or impeding optimal therapeutics? Fertil Steril. 2020;114:476-82.
7. Rao PK, Boulet SL, Mehta A, Hotaling J, Eisenberg ML, Honig SC, et al. Trends in testosterone replacement therapy use from 2003 to 2013 among reproductiveage men in the United States. J Urol. 2017;197:1121-6.

8. Rodriguez Socarrás M, Loeb S, Teoh JY, Ribal MJ, Bloemberg J, Catto J, et al. Telemedicine and smart working: recommendations of the European Association of Urology. Eur Urol. 2020;78:812-9.

9. Nangia AK, Likosky DS, Wang D. Distribution of male infertility specialists in relation to the male population and assisted reproductive technology centers in the United States. Fertil Steril. 2010;94:599-609.

10. Houman JJ, Eleswarapu SV, Mills JN. Current and future trends in men's health clinics. Transl Androl Urol. 2020;9:S116-S22.

11. Mulhall JP, Trost LW, Brannigan RE, Kurtz EG, Redmon JB, Chiles KA, et al. Evaluation and management of testosterone deficiency: AUA guideline. J Urol. 2018;200:423-32.

12. Office of Healthcare Inspections. Testosterone replacement therapy initiation and follow-up evaluation in VA male patients. 2018. https://www.va.gov/oig/pubs/ VAOIG-15-03215-154.pdf.

13. Lynch L, Long M, Moorhead A. Young men, help-seeking, and mental health services: exploring barriers and solutions. Am J Mens Health. 2018;12:138-49.

14. McCreary DR, Oliffe JL, Black N, Flannigan R, Rachert J, Goldenberg SL. Canadian men's health stigma, masculine role norms and lifestyle behaviors. Health Promot Int. 2020;35:535-43.

15. Medina-Perucha L, Yousaf $O$, Hunter MS, Grunfeld EA. Barriers to medical helpseeking among older men with prostate cancer. J Psychosoc Oncol. 2017;35:531-43.

16. Galdas PM, Cheater F, Marshall P. Men and health help-seeking behaviour: literature review. J Adv Nurs. 2005;49:616-23.

17. Shanafelt TD, Hasan O, Dyrbye LN, Sinsky C, Satele D, Sloan J, et al. Changes in burnout and satisfaction with work-life balance in physicians and the general US working population between 2011 and 2014. Mayo Clin Proc. 2015;90:1600-13.

18. Nauheim J, North AC. An updated review on physician burnout in urology. Urol Clin North Am. 2021;48:173-8.

19. West CP, Dyrbye LN, Shanafelt TD. Physician burnout: contributors, consequences and solutions. J Intern Med. 2018;283:516-29.

20. Demaerschalk BM, Cassivi SD, Blegen RN, Borah B, Moriarty J, Gullerud R, et al. Health economic analysis of postoperative video telemedicine visits to patients' homes. Telemed J E Health. 2021;27:635-40.

21. Zholudev V, Safir IJ, Painter MN, Petros JA, Filson CP, Issa MM. Comparative cost analysis: teleurology vs conventional face-to-face clinics. Urology 2018;113:40-4.

22. Zakaria A, Miclau TA, Maurer T, Leslie KS, Amerson E. Cost minimization analysis of a teledermatology triage system in a managed care setting. JAMA Dermatol. 2021;157:52-8.

23. Portney DS, Ved R, Nikolian V, Wei A, Buchmueller T, Killaly B, et al. Understanding the cost savings of video visits in outpatient surgical clinics. Mhealth 2020;6:32.

24. Ramaswamy A, Yu M, Drangsholt S, Ng E, Culligan PJ, Schlegel PN, et al. Patient satisfaction with telemedicine during the COVID-19 pandemic: retrospective cohort study. J Med Internet Res. 2020;22:e20786. 
25. Chu S, Boxer R, Madison P, Kleinman L, Skolarus T, Altman L, et al. Veterans affairs telemedicine: bringing urologic care to remote clinics. Urology 2015;86:255-60.

26. Margolin EJ, Pina Martina LA, Miles CH, Wenske S, McKiernan JM, DeCastro GJ, et al. Telemedicine in management of genitourinary malignancies: patient and physician perspectives. Urol Oncol. 2021;39:480-6.

27. Gan Z, Lee SY, Weiss DA, Van Batavia J, Siu S, Frazier J, et al. Single institution experience with telemedicine for pediatric urology outpatient visits: adapting to COVID-19 restrictions, patient satisfaction, and future utilization. J Pediatr Urol. 2021;17:480.e1.

28. Giusto LL, Derisavifard S, Zahner PM, Rueb JJ, Deyi L, Jiayi L, et al. Telemedicine follow-up is safe and efficacious for synthetic midurethral slings: a randomized, multi-institutional control trial. Int Urogynecol J. 2021;1-9.

29. Chesnel C, Hentzen C, Le Breton F, Turmel N, Tan E, Haddad R, et al. Efficiency and satisfaction with telephone consultation of follow-up patients in neuro-urology: experience of the COVID-19 pandemic. Neurourol Urodyn. 2021;40:929-37.

30. Shiff B, Frankel J, Oake J, Blachman-Braun R, Patel P. Patient satisfaction with telemedicine appointments in an academic andrology-focused urology practice during the COVID-19 pandemic. Urology. 2021;153:35-41.

31. Group TC-HCTISW. Telehealth impact: physician survey analysis. 2020. https:// telehealth-c19hcc-org-bzh6faksvq-uk.a.run.app/telehealth/physician-survey-analysis/? mc_id =us\&utm_source=newsnetwork\&utm_medium $=\mid$ \&utm_content $=$ content\&utm_campaign $=$ mayoclinic\&geo=national\&placementsite=enterprise $\&$ cauid $=100721$.

32. Viers BR, Lightner DJ, Rivera ME, Tollefson MK, Boorjian SA, Karnes RJ, et al. Efficiency, satisfaction, and costs for remote video visits following radical prostatectomy: a randomized controlled trial. Eur Urol. 2015;68:729-35.

33. Blandford A, Wesson J, Amalberti R, AlHazme R, Allwihan R. Opportunities and challenges for telehealth within, and beyond, a pandemic. Lancet Glob Health. 2020;8:e1364-e5.

34. Mishori R, Antono B. Telehealth, rural america, and the digital divide. J Ambul Care Manag. 2020;43:319-22.

35. Kim DW, Choi JY, Han KH. Risk management-based security evaluation model for telemedicine systems. BMC Med Inf Decis Mak. 2020;20:106.

36. Halpern JA, Brannigan RE. Testosterone deficiency. JAMA. 2019;322:1116.

37. Yeo S, Holl K, Peñaherrera N, Wissinger U, Anstee K, Wyn R. Burden of male hypogonadism and major comorbidities, and the clinical, economic, and humanistic benefits of testosterone therapy: a narrative review. Clinicoecon Outcomes Res. 2021;13:31-8.

38. Rastrelli G, Corona G, Maggi M. Both comorbidity burden and low testosterone can explain symptoms and signs of testosterone deficiency in men consulting for sexual dysfunction. Asian J Androl. 2020;22:265-73.

39. Mitchell R, Thomas SD, Langlois NE. How sensitive and specific is urinalysis 'dipstick' testing for detection of hyperglycaemia and ketosis? An audit of findings from coronial autopsies. Pathology 2013;45:587-90.

40. Shamsham F, Mitchell J. Essentials of the diagnosis of heart failure. Am Fam Physician. 2000;61:1319-28.

41. Burnett AL, Nehra A, Breau RH, Culkin DJ, Faraday MM, Hakim LS, et al. Erectile dysfunction: AUA guideline. J Urol. 2018;200:633-41.

42. Shahinyan RH, Amighi A, Carey AN, Yoffe DA, Hodge DC, Pollard ME, et al. Directto-consumer internet prescription platforms overlook crucial pathology found during traditional office evaluation of young men with erectile dysfunction. Urology. 2020;143:165-72.

43. Zhou CK, Advani S, Chaloux M, Gibson JT, Yu M, Bradley M, et al. Trends and patterns of testosterone therapy among U.S. male medicare beneficiaries, 1999 to 2014. J Urol. 2020;203:1184-90.

44. Carter HB, Albertsen PC, Barry MJ, Etzioni R, Freedland SJ, Greene KL, et al. Early detection of prostate cancer: AUA guideline. J Urol. 2013;190:419-26.

45. Kelm RC, Adotama P, Stratton JS, Levin JI. Testosterone pellet-induced generalized drug eruption. Cutis. 2020;106:E15-E6.

46. Andino JJ, Guduguntla V, Weizer A, Roberts WW, Wittmann D, Miller D, et al. Examining the value of video visits to patients in an outpatient urology clinic. Urology. 2017;110:31-5.

47. Morden NE, Woloshin S, Brooks CG, Schwartz LM. Trends in testosterone prescribing for age-related hypogonadism in men with and without heart disease. JAMA Intern Med. 2019;179:446-8.
48. Drake C, Zhang Y, Chaiyachati KH, Polsky D. The limitations of poor broadband internet access for telemedicine use in rural america: an observational study. Ann Intern Med. 2019;171:382-4.

49. Perrin A. Digital gap between rural and nonrural America persists: Pew Research Center. 2019. https://www.pewresearch.org/fact-tank/2019/05/31/digital-gapbetween-rural-and-nonrural-america-persists/.

50. Kemp MT, Liesman DR, Williams AM, Brown CS, lancu AM, Wakam GK, et al. Surgery provider perceptions on telehealth visits during the COVID-19 pandemic: room for improvement. J Surg Res. 2021;260:300-6.

51. Black Book Research. Healthcare data breaches costs industry $\$ 4$ billion by year's end, 2020 will be worse reports new black book survey. 2020. https://www. prnewswire.com/news-releases/healthcare-data-breaches-costs-industry-4billion-by-years-end-2020-will-be-worse-reports-new-black-book-survey300950388.html. Accessed 3 May 2021.

52. Maji A, Mukhoty A, Majumdar A, Mukhopadhyay J, Sural S, Paul S. Security analysis and implementation of web-based telemedicine services with a four-tier architecture. 2008 Second International Conference on Pervasive Computing Technologies for Healthcare; 2008. p. 46-54.

53. She H, Lu Z, Jantsch A, Zheng L, Zhou D. A network-based system architecture for remote medical applications. Network Research Workshop; 2007.

54. Betancourt JA, Rosenberg MA, Zevallos A, Brown JR, Mileski M. The impact of COVID-19 on telemedicine utilization across multiple service lines in the United States. Healthcare. 2020;8:380.

55. Fields BG. Regulatory, legal, and ethical considerations of telemedicine. Sleep Med Clin. 2020;15:409-16.

56. Kamsu-Foguem B, Foguem C. Telemedicine and mobile health with integrative medicine in developing countries. Health Policy Technol. 2014;3:264-71.

57. Nittari G, Khuman R, Baldoni S, Pallotta G, Battineni G, Sirignano A, et al. Telemedicine practice: review of the current ethical and legal challenges. Telemed J E Health. 2020;26:1427-37.

58. Association UFaD. FDA approves new changes to testosterone labeling regarding the risks associated with abuse and dependence of testosterone and other anabolic androgenic steroids (AAS). 2016. https://www.fda.gov/drugs/drugsafety-and-availability/fda-approves-new-changes-testosterone-labelingregarding-risks-associated-abuse-and-dependence.

59. List of services payable under the medicare physician fee schedule when furnished via telehealth. The Centers for Medicare and Medicaid Services. 2021. https://www.cms.gov/Medicare/Medicare-General-Information/Telehealth/ Telehealth-Codes.

60. Hare N, Bansal P, Bajowala SS, Abramson SL, Chervinskiy S, Corriel R, et al. Work group report: COVID-19: unmasking telemedicine. J Allergy Clin Immunol Pract. 2020;8:2461-73.e3.

\section{AUTHOR CONTRIBUTIONS}

JMD designed the work, acquired data, drafted and revised the manuscript, approved the final version, and agreed to be accountable for all aspects of the work in ensuring that questions related to the accuracy or integrity of any part of the work are appropriately investigated and resolved. RJF acquired data, drafted and revised the manuscript, and approved the final version. JAH designed the work drafted and revised the manuscript and approved the final version

\section{COMPETING INTERESTS}

The authors declare no competing interests.

\section{ADDITIONAL INFORMATION}

Correspondence and requests for materials should be addressed to Justin M. Dubin.

Reprints and permission information is available at http://www.nature.com/ reprints

Publisher's note Springer Nature remains neutral with regard to jurisdictional claims in published maps and institutional affiliations. 